\title{
Heterostructure ohmic contacts to p-CdTe polycrystalline films
}

\author{
A.V. Sukach ${ }^{1}$, V.V. Tetyorkin ${ }^{1}$, A.I. Tkachuk ${ }^{2}$ \\ ${ }^{I}$ V. Lashkaryov Institute of Semiconductor Physics, NAS of Ukraine, \\ 41, prospect Nauky, 03028 Kyiv, Ukraine \\ ${ }^{2}$ V. Vinnichenko Kirovograd State Pedagogical University, Kirovograd, Ukraine \\ Phone: 38 (044) 525-54-61, e-mail: teterkin@isp.kiev.ua
}

\begin{abstract}
Heterostructure contacts $p^{+}-\mathrm{PbTe} / p-\mathrm{CdTe}$ were prepared using the hot-wall technique on glassceramic substrates. It has been shown that the potential barrier at the $p^{+}-\mathrm{PbTe} / p$-CdTe interface is not formed in the case of heavily doped lead telluride. That allows one to create ohmic heterocontacts of metal- $p^{+}-\mathrm{PbTe} / p$-CdTe type. The transverse and in-plane transport of carriers has been investigated as a function of bias voltage and temperature. The current-voltage characteristics measured for the transverse arrangement of contacts exhibited ohmic behavior. The current-voltage characteristics of these contacts are determined by unipolar injection of holes from $p^{+}$-PbTe into $p$-CdTe. The inplane transport has been explained by presence of potential barriers at the grain boundaries. The potential barrier height has been estimated to be $\sim 0.1 \mathrm{eV}$ at room temperature. The mechanism of carrier transport is thermionic emission.
\end{abstract}

Keywords: polycrystalline $\mathrm{CdTe}$, transverse and in-plane transport, ohmic contact, $\mathrm{PbTe}$.

Manuscript received 07.02.14; revised version received 02.07.14; accepted for publication 16.09.14; published online 30.09.14.

\section{Introduction}

CdTe thin films are widely used for manufacturing different semiconductor devices such as solar cells, infrared, X-ray and gamma-ray detectors for medical and industrial imaging systems, etc. [1-3] Fabrication of ohmic contacts to CdTe-based devices with stable and reproducible characteristics still remains an important problem, especially for $p$-type material. As a rule, it is achieved by formation of heavily doped region at the film surface using appropriate dopant. For instance, copper-containing contacts have been developed for $p$ $\mathrm{CdTe}$, which include $\mathrm{Cu}_{2} \mathrm{Te}, \mathrm{Cu}-\mathrm{Au}$ alloy, $\mathrm{ZnTe}: \mathrm{Cu}$ and $\mathrm{HgTe}: \mathrm{Cu}$ [4-6]. By increasing $p$-type doping, copper provides good ohmic contacts. It must be pointed out that the highest reported efficiency of solar cells $(\sim 16.5 \%)$ is achieved in $\mathrm{CdS} / \mathrm{CdTe}$ heterostructures incorporated with copper-containing contacts [7]. The main drawback of these contacts is fast migration of copper in CdTe polycrystalline films. It is responsible for long-term degradation of sollar cell performances. Because of this reason, copper-free contacts have attracted great interest in CdTe technology. Several solutions were earlier proposed including use of different materials, such as $\mathrm{Sb}_{2} \mathrm{Te}_{3}$ and $\mathrm{Ni}_{2} \mathrm{P}$ [8-10].

The purpose of this study is to investigate $p^{+}-\mathrm{PbTe}$ heterostructure contacts to $p$-CdTe polycrystalline films. The main reasons for the choice of $p^{+}-\mathrm{PbTe}$ as a contact material are as follows: a) $p^{+}-\mathrm{PbTe} / p$-CdTe heterojunction is characterized by a small lattice constant mismatch $\sim 0.3 \%$; b) small band discontinuity in the valence band enables implementation of efficient transport of holes at low bias voltages [12-17]; c) growth technology of PbTe layers is well developed [18]; d) ohmic contacts to highly doped $p^{+}-\mathrm{PbTe}$ can be easily manufactured. 


\section{Samples and experimental techniques}

In this study, CdTe polycrystalline films were prepared by hot-wall epitaxy technique on glassceramic substrates. The substrates were etched in aqueous solution of hydrofluoric acid and washed in distilled water. Grain sizes were determined using the linear intercept method [19]. An undoped $p$-CdTe with the resistivity $(1 \ldots 2) \cdot 10^{3} \Omega \cdot \mathrm{cm}$ at room temperature was used for deposition of polycrystalline films with the average thickness close to $130 \mu \mathrm{m}$ and grain size $\sim 60 \mu \mathrm{m}$. The temperatures of evaporation source, substrate and wall were fixed at 600,410 , and $430{ }^{\circ} \mathrm{C}$, respectively. In order to investigate carrier transport mechanisms, the heterostructure contacts were manufactured growing the $p^{+}-\mathrm{PbTe}$ polycrystalline films at the surface of $p$-CdTe films. For this purpose, Tl-doped $p^{+}-\mathrm{PbTe}$ with the impurity concentration 0.8 at. $\%$ was used as the contact material. The hole concentration in this material was estimated to be of the order of $10^{19} \mathrm{~cm}^{-3}$ at room temperature. Finally, Au metal pads were deposited using electrolysis at the surface of $p^{+}-\mathrm{PbTe}$ films. The carrier transport mechanisms were investigated by measuring the current-voltage characteristics within the temperature range $218 \ldots 387 \mathrm{~K}$ and temperature dependences of dark current at fixed values of bias voltage within the same temperature range. In-plane and transverse arrangements of contacts were used. In the latter case, the investigated CdTe films with the thickness close to $120 \mu \mathrm{m}$ were sandwiched between two PbTe contact layers, grown using the same technique. The bottom and upper layers had the thickness 25 to $27 \mu \mathrm{m}$ and 10 to $15 \mu \mathrm{m}$, accordingly. In the in-plane arrangement, the distance between contacts was approximately $2 \mathrm{~mm}$.

\section{Results and discussion}

Typical current-voltage characteristics measured at different temperatures in the structures with transverse and in-plane arrangements of contacts are shown in Figs. 1 and 2, respectively. As seen, for the transverse arrangement, the characteristics are linear and do not depend on the polarity of applied voltage. In the case of in-plane arrangement, the current-voltage characteristics exhibited more complicate behavior (Fig. 2). At low bias voltages, the linear $I-U$ dependence is observed, followed by the sub-linear one at higher voltages. The non-linear branch of the current-voltage characteristic is fitted by a power-law dependence $I \sim U^{n}$, with the exponent value $n \approx 0.5$. The temperature dependences of the dark current measured at fixed bias voltages are shown in Figs. 3 and 4. For the transverse arrangement of contacts, experimental data were plotted in coordinates $\ln I-1 / T$ and represented by two linear branches. From their slopes, the activation energies $0.32 \mathrm{eV}$ at temperatures less than $222 \mathrm{~K}$ and $0.48 \mathrm{eV}$ at higher temperatures were determined. It should be pointed out that the determined energies do not depend on the applied voltage in the case of the transverse arrangement of contacts. However, in the case of the inplane arrangement, the dependence of the activation energy on applied voltage is observed. Namely, the activation energy increases from the value $0.58 \mathrm{eV}$ at biases 2 and $10 \mathrm{~V}$ to the value $0.69 \mathrm{eV}$ at the bias voltage $150 \mathrm{~V}$. Note also that these values exceed those determined for the transverse arrangement of contacts.

The carrier transport mechanisms in $\mathrm{CdTe}$ polycrystalline films are not fully understood. As in the case of other semiconductor polycrystalline films, two paths for the current flow are possible, namely: through the grains and along the grain boundaries. In the latter case, one would expect the carrier transport characteristics similar to those observed in disordered semiconductors [20]. In the investigated films composed of large grains, these characteristics were not observed. Since the $p^{+}-\mathrm{PbTe}$ contact layers have metallic conductivity, the determined values of the activation energies refer to the carrier transport through the grains (across potential barriers at the grain boundaries). The linear current-voltage characteristics observed for the transverse arrangement of contacts clearly indicate their ohmic nature as well as preferential transport of carriers through the grains, but not along the grain boundaries. Also, the high-frequency (1 MHz) capacitance of these structures is determined by contact geometry and does not depend on the polarity of bias voltage, indicating the absence of a potential barrier at the $p^{+}-\mathrm{PbTe} / p-\mathrm{CdTe}$ heterojuction. The temperature dependences shown in Fig. 3 are explained by thermal excitation of carriers from deep levels.

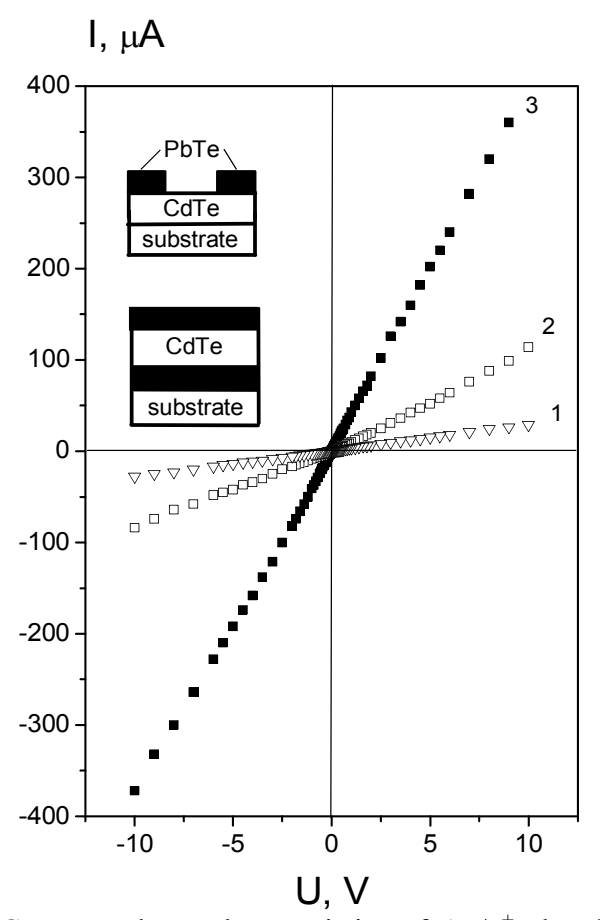

Fig. 1. Current-voltage characteristics of $\mathrm{Au} / p^{+}-\mathrm{PbTe} / p-\mathrm{CdTe}$ contacts for transverse arrangements of contacts at temperatures, K: $218(1), 329(2), 387(3)$. In-plane and transverse arrangements of contacts are also shown. 
$\mathrm{I}, \mu \mathrm{A}$

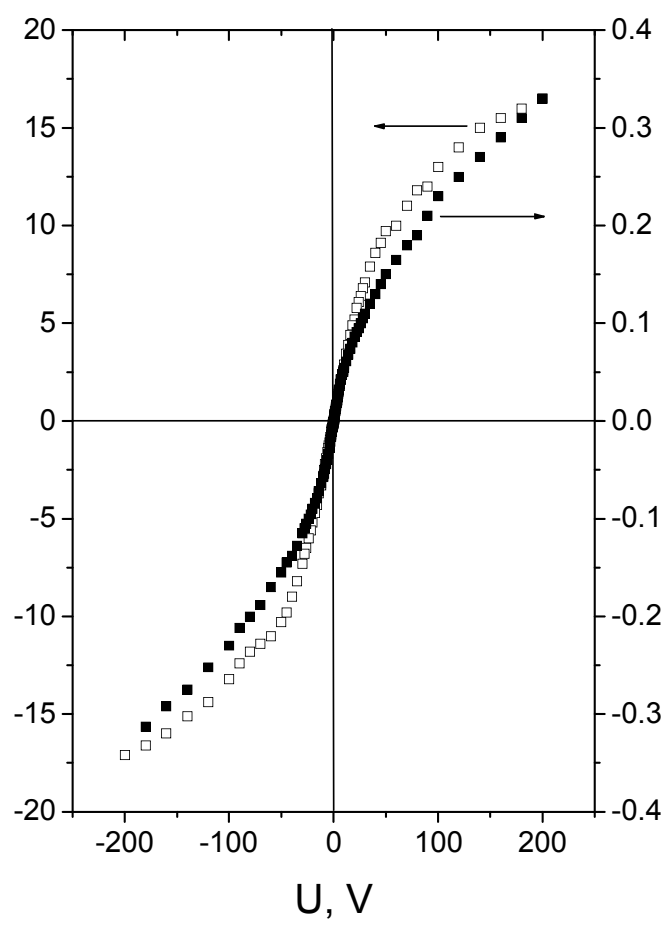

Fig. 2. In-plane current-voltage characteristics of contacts at $298 \mathrm{~K}$ (close dots) and $387 \mathrm{~K}$ (open dots).

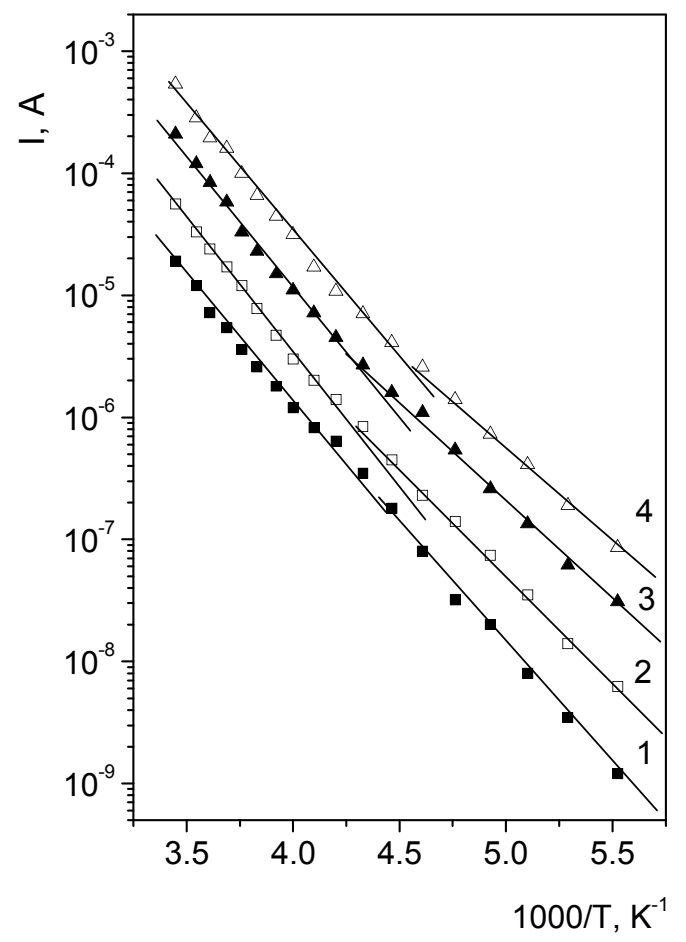

Fig. 3. Temperature dependences of the dark current for transverse arrangement of contacts at the bias voltages, V: $1(1), 4(2), 9(3), 18(4)$.

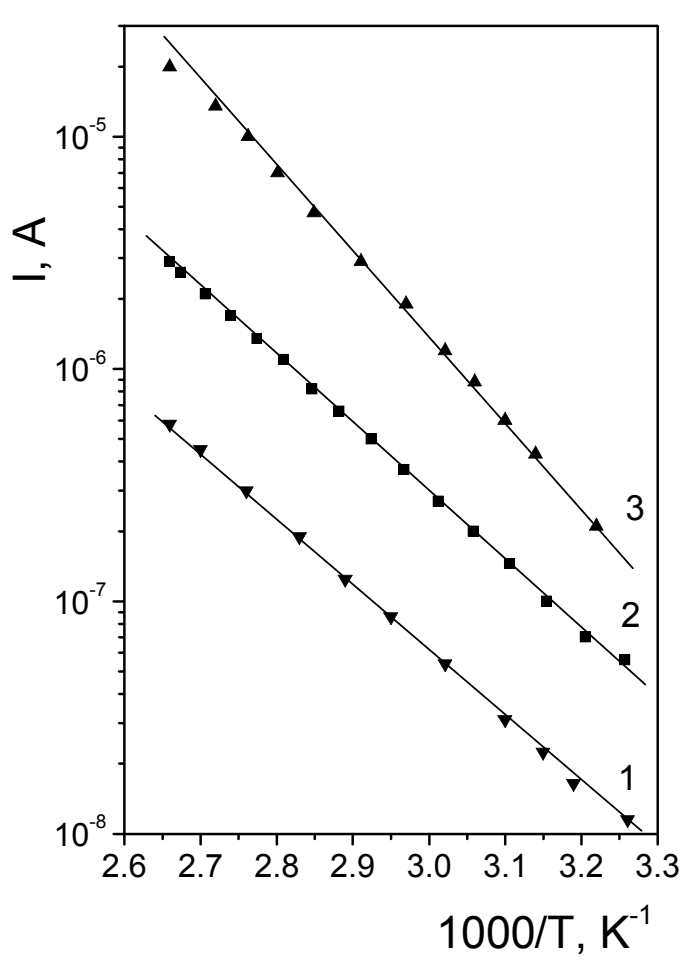

Fig. 4. Temperature dependences of the dark current for inplane arrangement of contacts at the bias voltages, V: $2(1)$, $10(2), 150(3)$.

In the case of in-plane arrangement of contacts, the shape of the observed $I-U$ dependences indicates the existance of potental barriers at the grain boundaries. Also, it should be pointed out a non-trivial dependence of the activation energy determined from the slope of the experimental curves in Fig. 4. The increase of the activation energy cannot be explained by Frenkel-Poole emission, since the ionization energy appearing in this case decreases with the applied bias. This decrease was observed, for instance, in the well-investigated $\mathrm{ZnO}$ polycrystalline films [21]. Also important is the fact that the activation energy of the dark current in lateral geometry measurements exceeds values obtained for transverse geometry. From this exceeding, the height of potential barriers at the grain boundaries can be estimated. In this case, the activation energy of the dark current is represented by the sum of the activation energy of defect levels inside the grain bulk and height of the potential barrier. The perfomed estimates of the potential barrier height give the value $0.2 \mathrm{eV}$ at zero temperature and $0.1 \mathrm{eV}$ at room temperature. The growth of the activation energy at the high bias voltage can be explained by its distribution between the grain bulk and potential barrier. At low bias voltages, the barrier height remains almost unchanged, thus the dark current is determined by the activation energy of defect levels in the bulk. At higher voltages, the activation energy is determined by the reversely biased barrier at the grain boundary. The width of the space charge region related 
to this barrier is comparable with the grain size or even exceeds it. It results in a tendency to saturation of $I-U$ curves shown in Fig. 2. For the determined values of the barrier height, the space charge region width is close to $32 \mu \mathrm{m}$ at zero bias and increases in the range from 45 to $270 \mu \mathrm{m}$ with the bias voltage increasing from 2 to $150 \mathrm{~V}$. These estimates were obtained for the hole mobility in the bulk of grains $10 \mathrm{~cm}^{2} / \mathrm{V} \cdot \mathrm{s}$, using the room temperature conductivity of CdTe films, measured in the transverse arrangement of contacts.

\section{Conclusions}

1. The current-voltage characteristics are investigated in $\mathrm{Au}(\mathrm{Sn}) / p^{+}-\mathrm{PbTe} / \mathrm{p}-\mathrm{CdTe}$ heterostructure contacts within the temperature range $218 \ldots 387 \mathrm{~K}$. For the transverse arrangement of contacts, the currentvoltage characteristics are linear within the range of bias voltages $\pm 10 \mathrm{~V}$. Ohmic current-voltage characteristics has been explained by monopolar injection of holes from the $p^{+}$-PbTe contact layer. The activation character of the temperature dependences for dark conductivity has been explained by thermal excitation of holes from the deep levels with the energies 0.32 and $0.48 \mathrm{eV}$.

2. The in-plane conductivity has activation character with the activation energy that exceeds the corresponding values in the transverse arrangement of contacts. This fact is due to the presence of potential barriers at grain boundaries in $p$-CdTe polycrystalline films. The barrier height has been estimated to be close to $0.2 \mathrm{eV}$ and $0.1 \mathrm{eV}$ at zero and room temperatures, respectively. The in-plane transport of carriers occurs due to the thermionic emission mechanism.

\section{References}

1. K.L. Chopra, S.R. Das, Thin Film Solar Cells. Plenum Press, New York, 1983.

2. T.M. Razykov, C.S. Ferekides, D. Morel, E. Stefanakos, H.S. Ullal, H.M. Upadhyaya, Solar photovoltaic electricity: Current status and future prospects // Solar Energy, 85(8), p. 1580-1608 (2011).

3. M. Hage-Ali and P. Siffert, Semiconductors for room temperature nuclear detector application // Semiconductor and Semimetals, Vol. 43, Eds. T.E. Schlesinger, and R.B. James. Academic Press, San Diego (1995).

4. K.D. Dobson, I. Visoly-Fisher, G. Hodes and D. Cahen, Stability of CdTe/CdS thin-film solar cells // Solar Energy Materials and Solar Cells, 62(3), p. 295-325 (2000).

5. T.L. Chu, S.S. Chu, Thin film II-VI photovoltaics // Solid-State Electron., 38(3), p. 533-549 (1995).

6. T.A. Gessert, S. Asher, S. Johnston, M. Young, P. Dippo, C. Corwine, Analysis of $\mathrm{CdS} / \mathrm{CdTe}$ devices incorporating a $\mathrm{ZnTe}: \mathrm{Cu} / \mathrm{Ti}$ contact // Thin Solid Films, 515(15), p. 6103-6106 (2007).
7. X. Wu, High-efficiency polycrystalline CdTe thinfilm solar cells // Solar Energy, 77, p. 803-814 (2004).

8. B. Ghosh, S. Purakayastha, P.K. Datta, R.W. Miles, M.J. Carter, R. Hill, Formation of a stable ohmic contact to CdTe thin films through the diffusion of P from Ni-P // Semicond. Sci. Tech., 10(1), p. 71-76 (1995).

9. N. Romeo, A. Bosio, R. Tedeschi, A. Romeo, V. Canevari, A highly efficient and stable CdTe/CdS thin film solar cell // Solar Energy Mat. Solar Cells, 58, p. 209-218 (1999).

10. D.L. Bätzner, A. Romeo, M. Terheggen, M. Döbeli, H. Zogg, A.N. Tiwari, Stability aspects in $\mathrm{CdTe}_{\mathrm{y}} \mathrm{CdS}$ solar cells // Thin Solid Films, 451-452, p. 536-543 (2004).

11. W. Walukiewicz, Mechanism of Fermi-level stabilization in semiconductors // Phys. Rev. B, 37, p. 4760-4763 (1988).

12. R. Leitsmann, L.E. Ramos and F. Bechstedt, Structural properties of $\mathrm{PbTe} / \mathrm{CdTe}$ interfaces from first principles // Phys. Rev. B, 74, p. 085309-1085309-8 (2006).

13. R. Leitsmann and F. Bechstedt, Electronic-structure calculations for polar lattice-structure-mismatched interfaces PbTe/CdTe (100) // Phys. Rev. B, 76, p. 125315-1-125315-11 (2007).

14. M. Bukala, P. Sankowski, R. Buczko and P. Kacman, Crystal and electronic structure of $\mathrm{PbTe} / \mathrm{CdTe}$ nanostructures // Nanoscale Res. Lett. 6, p. 126 (2011).

15. J. Si, S. Jin, H. Zhang, P. Zhu, D. Qiu, H. Wu, Experimental determination of valence band offset at $\mathrm{PbTe} / \mathrm{CdTe}(111)$ heterojunction interface by $\mathrm{x}-$ ray photoelectron spectroscopy // Appl. Phys. Lett. 93(20), p. 202101-1-202101-3 (2008).

16. C.F. Cai, H.Z. Wu, J.X. Si, S.Q. Jin, W.H. Zhang, $\mathrm{Y} \mathrm{Xu}$, Energy band alignment of PbTe/CdTe(111) interface determined by ultraviolet photoelectron spectra using synchrotron radiation // Chin. Phys. $B$, 19(7), p. 077301-1-077301-3 (2010).

17. V.V. Tetyorkin, A.V. Sukach, A.I. Tkachuk, S.P. Movchan, Injection current and infrared photosensitivity in iso-type p-PbTe/p-CdTe heterojunctions // Semiconductor Physics, Quantum Electronics and Optoelectronics, 16(1), p. 57-61 (2013).

18. G. Nimitz, B. Schlicht, Narrow-gap lead salts, in: Narrow-Gap Semiconductors. Springer, Berlin, 1985.

19. H. Abrams, Grain size measurement by the intercept method // Metallography, 4(1), p. 5978 (1971).

20. B.I. Shklovskii and A.L. Efros, Electronic Properties of Doped Semiconductors. Springer, Berlin, 1984.

21. M.A. Alim, S. Li, F. Liu, and P. Cheng, Electrical barriers in the $\mathrm{ZnO}$ varistor grain boundaries // Phys. status solidi (a), 203(2), p. 410-427 (2006). 